\title{
A pilot study investigating the effects of yakult fermented milk drink (L. casei Shirota) on salivary IFN- $\gamma$, sIgA, IgA1 and IgA2 in healthy volunteers
}

\author{
E. O'Connel, J. Allgrove, L. Pollard, M. Xiang and L. S. Harbige \\ School of Science, University of Greenwich at Medway, Chatham Maritime, Kent ME4 4TB, UK
}

This pilot study investigated the effects on salivary cytokine and $\operatorname{SIgA}$ before and after daily consumption of Lactobacillus casei Shirota $(\mathrm{LcS})$ in nine healthy adult volunteers. Subjects were asked to consume two bottles each day of a fermented milk drink containing a total of $1.3 \times 10^{10}$ live cells of LcS (Yakult), for 4 weeks. Volunteers were asked to avoid food and drink for at least $1 \mathrm{~h}$ before morning unstimulated saliva samples were collected over a 5 min period. Samples were collected at baseline, at week 1, 2 and 4 of LcS consumption, and 2 weeks after cessation of consumption. We found a significant transient increase in salivary IFN- $\gamma$ levels, but were unable to detect IL-12, and an increase in salivary sIgA, IgA1 and IgA2 secretion. Baseline salivary IFN- $\gamma$ was below the assay detection limits. However, at week 1, 4/9 subjects had detectable salivary IFN- $\gamma$ levels, $8 / 9(P<0.01)$ at week 2 and $3 / 9$ at week 4 . None had detectable IFN- $\gamma$ levels at week 6 . IFN- $\gamma$ concentrations ranged from 35.3 to $92.0 \mathrm{pg} / \mathrm{ml}$. There was a significant $(P<0.02)$ increase in salivary sIgA secretion rate at week 4 (Fig. A). At week 6 , the sIgA secretion rate was greater than baseline. The IgA1 secretion rate was significantly greater than baseline at week $4(P<0.05)$ and week $6(P<0.02)$ (Fig. B). The IgA2 secretion was also significantly increased at week $4(P<0.05)$ and week $6(P<0.01)$ (Fig. B) and the IgA1:IgA2 ratio significantly greater at week $2(P<0.01)$. This pilot study demonstrates that LcS can induce increased salivary IFN- $\gamma$, sIgA, IgA1 and IgA2 secretion in healthy adults, which may improve mucosal immunity in the upper respiratory tract and provide health related benefits. A larger detailed study investigating cytokine networks, immunoglobulins and $\mathrm{T}$ cell responses in different immune system compartments in parallel with a placebo control group would define more rigorously and precisely the immunological effects of $\mathrm{LcS}$ (Yakult).

Figure A

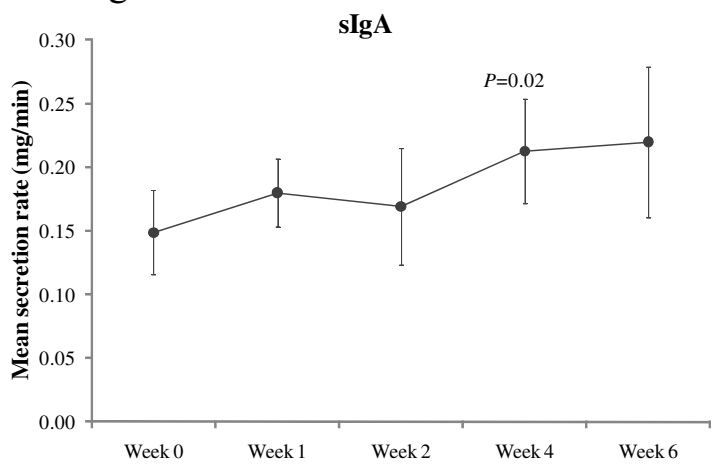

Figure B

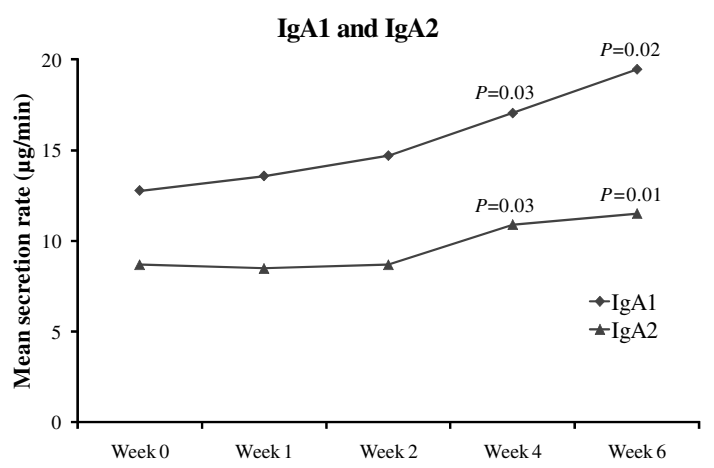

